\title{
Artificial E-region field-aligned plasma irregularities generated at pump frequencies near the second electron gyroharmonic
}

\author{
D. L. Hysell and E. Nossa \\ Earth and Atmospheric Science, Cornell University, Ithaca, NY, USA
}

Received: 2 March 2009 - Revised: 15 June 2009 - Accepted: 23 June 2009 - Published: 7 July 2009

\begin{abstract}
E region ionospheric modification experiments have been performed at HAARP using pump frequencies about $50 \mathrm{kHz}$ above and below the second electron gyroharmonic frequency. Artificial $\mathrm{E}$ region field-aligned plasma density irregularities (FAIs) were created and observed using the imaging coherent scatter radar near Homer, Alaska. Echoes from FAIs generated with pump frequencies above and below $2 \Omega_{e}$ did not appear to differ significantly in experiments conducted on summer afternoons in 2008, and the resonance instability seemed to be at work in either case. We argue that upper hybrid wave trapping and resonance instability at pump frequencies below the second electron gyroharmonic frequency are permitted theoretically when the effects of finite parallel wavenumbers are considered. Echoes from a sporadic $\mathrm{E}$ layer were observed to be somewhat weaker when the pump frequency was $50 \mathrm{kHz}$ below the second electron gyroharmonic frequency. This may indicate that finite parallel wavenumbers are inconsistent with wave trapping in thin sporadic E ionization layers.
\end{abstract}

Keywords. Radio science (Waves in plasma) - Space plasma physics (Active perturbation experiments; Waves and instabilities)

\section{Introduction}

The production of magnetic field-aligned plasma density irregularities (FAIs) is a signature feature of RF ionospheric modification experiments. Visible to coherent scatter radars, the irregularities provide a means of diagnosing the experiments with ground-based remote sensing. A number of radar observations have been conducted in concert with ionospheric heating experiments in an attempt to elucidate the

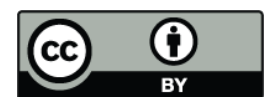

Correspondence to: D. L. Hysell (dlh37@cornell.edu) mechanisms responsible for creating the FAIs and other related phenomena (see reviews by Robinson, 1989; Frolov et al., 1997; Gurevich, 2007). While questions remain, the finding that FAIs are produced near the upper hybrid resonance level in the plasma rather than at the critical height (e.g. Djuth et al., 1985; Noble et al., 1987) focuses attention on two closely related instability processes. One of these is the thermal parametric instability (Grach et al., 1978; Das and Fejer, 1979; Fejer, 1979; Kuo and Lee, 1982; Dysthe et al., 1983; Mjølhus, 1990), and the other is the resonance instability (Vas'kov and Gurevich, 1977; Inhester et al., 1981; Grach et al., 1981; Dysthe et al., 1982; Lee and Kuo, 1983; Mjølhus, 1993).

In the thermal parametric instability, or the thermal oscillating two-stream instability (OTSI) more precisely, the pump electromagnetic wave is resonantly converted in the presence of field-aligned plasma density irregularities into upper hybrid waves which heat the plasma collisionally and, in turn, intensify the irregularities through differential thermal forcing. If the amplitude of the irregularities (striations) becomes sufficiently large, upper hybrid waves may be trapped within them, causing additional mode conversion, heating, and trapping in tandem with the formation of thermal cavitons. This is the condition for the resonance instability, which is characterized by explosive wave growth. Coherent scatter is then indicative of the meter-scale striations (e.g. Stubbe, 1996).

The behavior of upper-hybrid waves depends strongly on the frequency with respect to harmonics of the electron gyrofrequency, $n \Omega_{e}$, mainly because of sharply increased collisionless damping at these frequencies. A number of closely related phenomena, including stimulated electromagnetic emission, electron and ion heating, optical emissions, and irregularity generation are also affected or interrupted when pumping at or near gyroharmonic frequencies (see Kosch et al., 2005; Mishin et al., 2005 for experimental and theoretical summaries). By investigating the kinetic dispersion

Published by Copernicus Publications on behalf of the European Geosciences Union. 
relation for upper hybrid/ electron Bernstein waves, Mjølhus (1993) argued that striation formation should be suppressed at heating frequencies near $n \Omega_{e}$ for $n \geq 3$. Huang and Kuo (1994), Istomin and Leyser (2003), and Gurevich (2007) reached similar conclusions and also predicted that the suppression should be asymmetric, extending to frequencies further below the gyroharmonic frequency than above it. Direct evidence for this phenomenon in the $\mathrm{F}$ region came first in the form of reduced anomalous absorption and subsequent intensification of processes occurring above the upper hybrid resonance height (Honary et al., 1999) and then in the form of reduced coherent scatter measured at Sura and by SuperDARN (Ponomarenko et al., 1999; Kosch et al., 2002). Additional, albeit indirect, evidence came from optical observations of artificial airglow and inferences about upper-hybrid wave-induced electron acceleration (e.g. Kosch et al., 2005).

The situation for pump frequencies near the second electron gyroharmonic appears to be more complicated and could not be explored experimentally from the mid-1980s until recently with the advent of a low-frequency heating capability at HAARP. Coherent scatter is observed for pump frequencies near $2 \Omega_{e}$, and enhancements have been reported at frequencies just above $2 \Omega_{e}$ (Fialer, 1974; Minkoff et al., 1974; Kosch et al., 2007). Airglow intensifications are also reliably observed for pump frequencies slightly above $2 \Omega_{e}$ (Haslett and Megill, 1974; Djuth et al., 2005; Kosch et al., 2005). On the theoretical side, the threshold for excitation of thermal parametric instability can be shown to be reduced in the vicinity of the second electron gyroharmonic frequency (Grach, 1979), explaining the observed, aforementioned overshoot.

However, analysis also suggests that wave trapping may be prohibited entirely at pump frequencies below the second electron gyroharmonic frequency (Mjølhus, 1993; Huang and Kuo, 1994). The prediction has especially important implications for artificial $\mathrm{E}$ region FAIs, which are routinely and often necessarily (in view of the low E-region critical frequency) generated using pump frequencies below and often well below $2 \Omega_{e}$ (e.g. Fialer, 1974; Hysell, 2008). For example, the FAIs observed by Nossa et al. (2009) were mainly generated using a pump frequency of just $2.75 \mathrm{MHz}$. Theory in this area has mainly concentrated on F region applications, but renewed interest in E region heating experiments prompts reinvestigation of the experimental record and the underlying physics.

In this paper, we investigate the generation of FAIs in the E region ionosphere at pump frequencies above and below the second electron gyroharmonic. The frequencies used are insufficiently close (e.g. see Gurevich, 2007) to $2 \Omega_{e}$ to evaluate upper hybrid wave suppression and asymmetry and are instead widely separated to ensure bracketing of the second gyroharmonic under a variety of $\mathrm{E}$ region conditions. E region experiments offer some practical advantages from the diagnostic point of view. Using VHF radar, high-latitude artificial E region FAIs can be observed with relatively little reliance on refraction to satisfy the Bragg scattering condition and correspondingly little ambiguity with regard to echolocation. Likewise, E region echo Doppler shifts are generally small enough to render the target underspread, permitting unambiguous spectral estimation using conventional pulse-to-pulse signal processing. Furthermore, the daytime $\mathrm{E}$ region ionosphere is relatively consistent and invariant on a day-to-day and minute-to-minute basis by comparison to the $\mathrm{F}$ region, affording more possibilities for controlled experiments. However, the critical frequency in the high-latitude $\mathrm{E}$ region is seldom more than about $3 \mathrm{MHz}$, making it difficult to explore gyroharmonics with $n \geq 3$. Sporadic E layers can be utilized for observations at higher pump frequencies, but only at the expense of the controlled experiment aspect of the observations.

\section{Observations}

The coherent scatter radar used for this investigation is located at the NOAA Kasitsna Bay Laboratory near Seldovia, Alaska, approximately $470 \mathrm{~km}$ southwest of HAARP. It operates at $29.8 \mathrm{MHz}$ using a $12 \mathrm{~kW}$ peak power transmitter and two side-by-side transmitting antenna arrays with a combined gain of approximately $18.5 \mathrm{dBi}$. For the experiments described here, 13-bit Barker-coded pulses with a $10 \mu$ s baud width and a pulse repetition frequency (PRF) of $405 \mathrm{~Hz}$ were used, giving a transmitter duty cycle of $5.3 \%$ and a maximum unambiguously resolvable Doppler shift of just over $1000 \mathrm{~m} / \mathrm{s}$. Reception is performed using 6 spaced antenna arrays, providing 15 approximately coplanar, nonredundant baselines for interferometry. Additional details about the system and some of the diagnostics it can perform were given by Hysell (2008).

The nominal electron gyrofrequency, $\Omega_{e}$, in the E region over HAARP is precisely $1.5 \mathrm{MHz}(\sim 1.511 \mathrm{MHz}$ at $105 \mathrm{~km}$ altitude according to IGRF-2005). The E region critical frequency meanwhile typically reached $3 \mathrm{MHz}$ at midday in the summer of 2008, with an associated peak upper-hybrid frequency of $3.35 \mathrm{MHz}$. This made it possible to use heating frequencies above and below the second electron gyroharmonic frequency, $2 \Omega_{e}$, during the summer months, even during solar minimum, with the expectation of meeting the upper-hybrid resonance condition in the $\mathrm{E}$ region.

Attempts to generate E region field-aligned plasma density irregularities over HAARP using O-mode zenith heating at pump frequencies about $50 \mathrm{kHz}$ above and below the second electron gyroharmonic frequency were made at midday on 28 and 29 July 2008, during the Polar Aeronomy and Radio Science (PARS) Summer School period. The frequencies $2.945 \mathrm{MHz}$ and $3.045 \mathrm{MHz}$ were available for these experiments. Heating was performed using the full power of the HAARP facility with zenith pointing and $\mathrm{CW}$ modulation following a 1-min on, 1-min off pattern. Results for 28 July are shown in Fig. 1. 

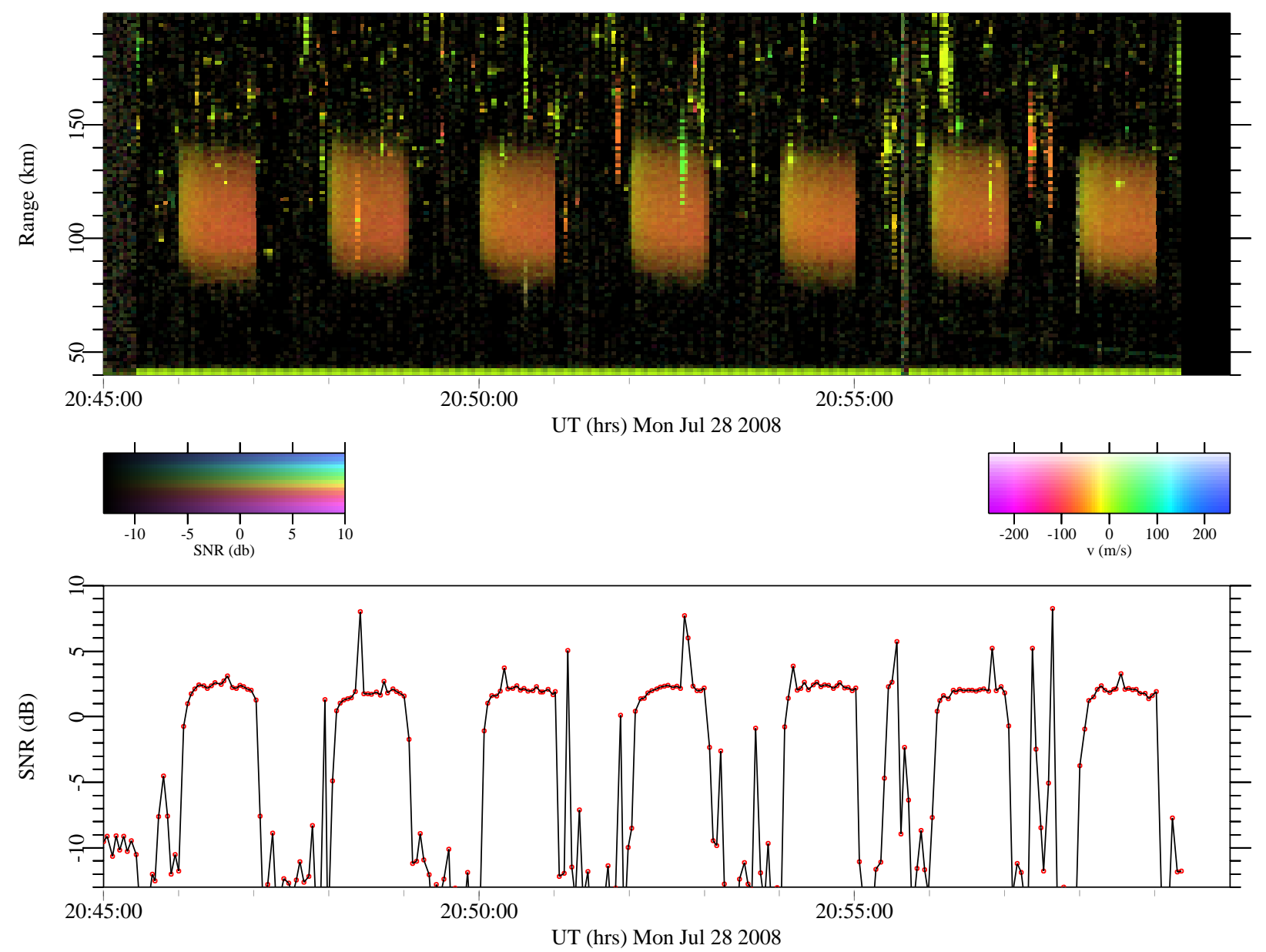

Fig. 1. Range-time Doppler intensity (RTDI) plot of backscatter from artificial E region FAIs over HAARP observed on 28 July 2008 . Here, the brightness, hue, and saturation of the pixels denote echo signal-to-noise ratio (SNR), Doppler shift, and spectral width. Note that the echoes from heater-induced FAIs are range aliased and that their true range is greater than their apparent range by $370 \mathrm{~km}$. The average signal-to-noise ratio for apparent ranges between $80-140 \mathrm{~km}$ is plotted beneath the RTDI plot. Echoes from meteor trails are also visible in the figure. The incoherent integration time for the figure is about $3 \mathrm{~s}$. Note that specular and long-lived meteor echoes are also visible throughout the figure.

In Fig. 1, Homer radar data are portrayed in range-timeDoppler-intensity (RTDI) format, in which the brightness, hue, and intensity of the pixels represent the signal-to-noise ratio, Doppler shift, and spectral width of the echoes according to the legend shown. Coherent integration by a factor of 4 was applied to these data prior to detection and spectral analysis. The echoes have Doppler shifts of about $-50 \mathrm{~m} / \mathrm{s}$ (red shifts) and RMS half-widths of about $50 \mathrm{~m} / \mathrm{s}$ in this case. The lower panel of the figure shows the average signal-to-noise ratio of echoes between $80-140 \mathrm{~km}$ apparent range (450$510 \mathrm{~km}$ true range). The incoherent integration time for the data in the figure is approximately $3 \mathrm{~s}$.

For this experiment, the pump frequency was $2.945 \mathrm{MHz}$ (3.045 MHz) during the 1st, 3rd, 5th, and 7th (2nd, 4th, and 6th) heating events depicted in Fig. 1. The echoes associated with the lower heating frequency are slightly stronger
$(<1 \mathrm{~dB})$ than at the higher frequency, despite the fact that the HAARP antenna has slightly higher gain $(\sim 0.3 \mathrm{~dB})$ at the higher frequency. The echoes for the lower frequency also appear to fall at slightly shorter ranges than for the higher frequency, something that could be indicative of a small difference in scattering altitude. The Doppler spectra for the two heating frequencies are indistinguishable.

Data from a short segment of an identical experiment performed on 29 July 2008, are shown in Fig. 2 (the complete dataset, typified by this short segment, appears in Fig. 1 of Nossa et al., 2009). The echoes received this time had very small Doppler shifts, and the data in the figure were coherently integrated by a factor of 16 prior to detection to improve the overall signal-to-noise ratio. The pump frequencies for the first and second heating event were 2.945 and $3.045 \mathrm{MHz}$, respectively. As in the previous example, the 

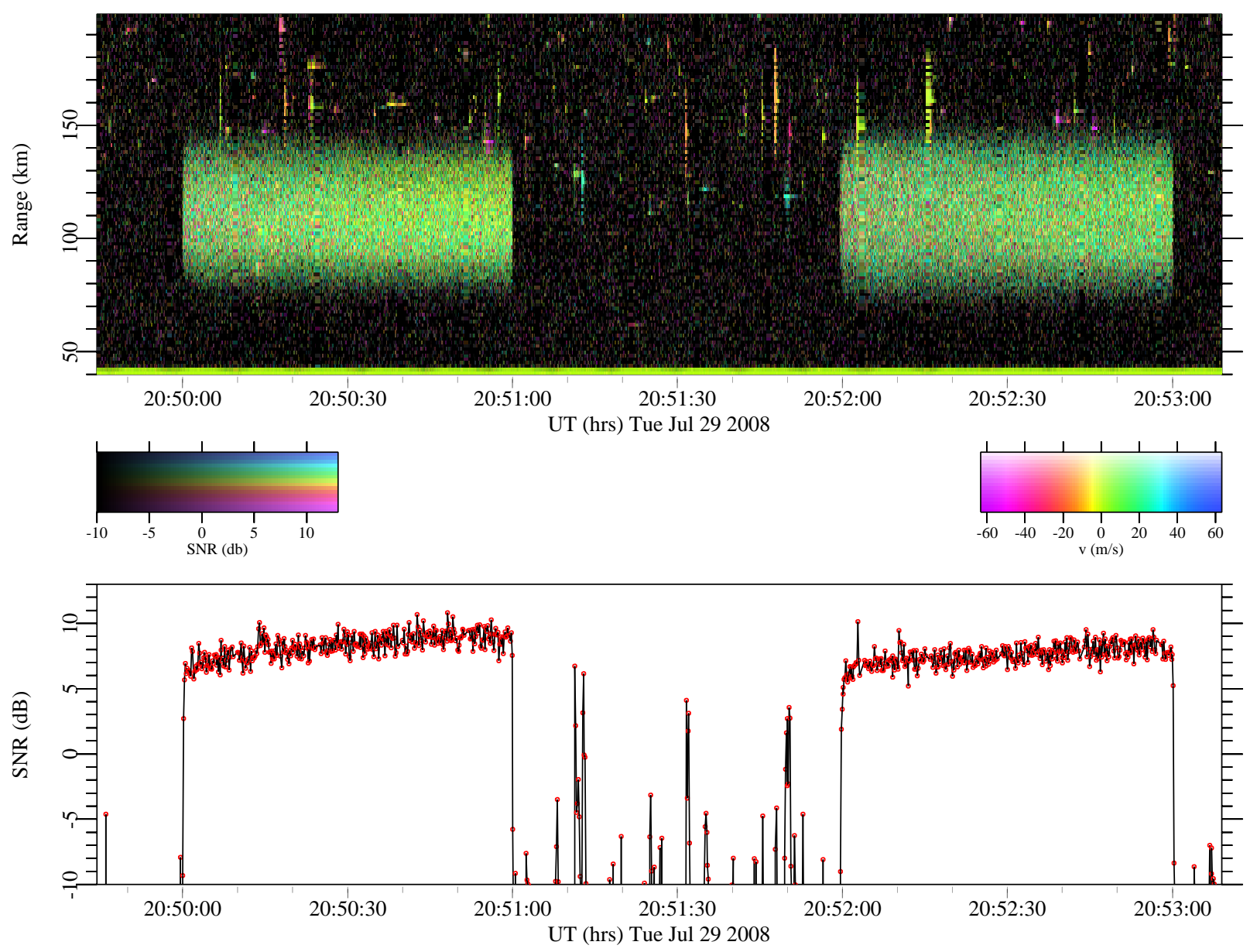

Fig. 2. RTDI plot of backscatter from artificial E region FAIs over HAARP observed on 29 July 2008. The incoherent integration time for the figure is about $0.158 \mathrm{~s}$.

echoes observed here were slightly stronger at the lower heating frequency. Note that this trend cannot be extrapolated; heating experiments conducted just prior to those shown here at a frequency of $2.75 \mathrm{MHz}$ produced coherent echoes with signal-to-noise ratios comparable to those generated at $2.945 \mathrm{MHz}$. Echoes from the higher heating frequency also extend over a somewhat broader span of ranges than echoes from the lower frequency, something that could be indicative of a larger modified volume and a higher scattering altitude at the higher frequency. Close examination of the relatively high time resolution data in Fig. 2 shows that the rise and fall times for the echoes at the two heating frequencies are, like the Doppler spectra, indistinguishable.

Additionally, an experiment similar to the preceding two was performed on 29 February 2008, prior to local midnight during active auroral conditions when a dense (critical frequency FoEs $\sim 4 \mathrm{MHz}$ ), blanketing sporadic E layer was over HAARP. Figure 3 shows the results in RTDI format. Natural auroral echoes are evident after about 08:15 UT. The riometer at HAARP indicated significant absorption af- ter about 08:00 UT but little in the preceding hour. Very weak echoes from artificially generated FAIs were also detected. These echoes had relatively large Doppler shifts of about $150 \mathrm{~m} / \mathrm{s}$ (blue shifts) and RMS spectral half widths of about $100 \mathrm{~m} / \mathrm{s}$. As in the previous examples, the heating frequency alternated between 2.945 and $3.045 \mathrm{MHz}$, with minute-long heating intervals at the higher frequency beginning at 07:44, 07:48, 07:52, 07:56, 08:00, and 08:04 UT and interleaving lower-frequency heating intervals. In this case, the echoes received appear to be stronger at the higher heating frequency by about $3-5 \mathrm{~dB}$. Low backscatter intensity and significant background variability both undermine the clarity of this result to some extent, however.

\section{Analysis}

The most significant finding from the July 2008 experiments described above is that there appear to be no significant differences between FAIs generated with pump frequencies about $50 \mathrm{kHz}$ above and below the second electron 

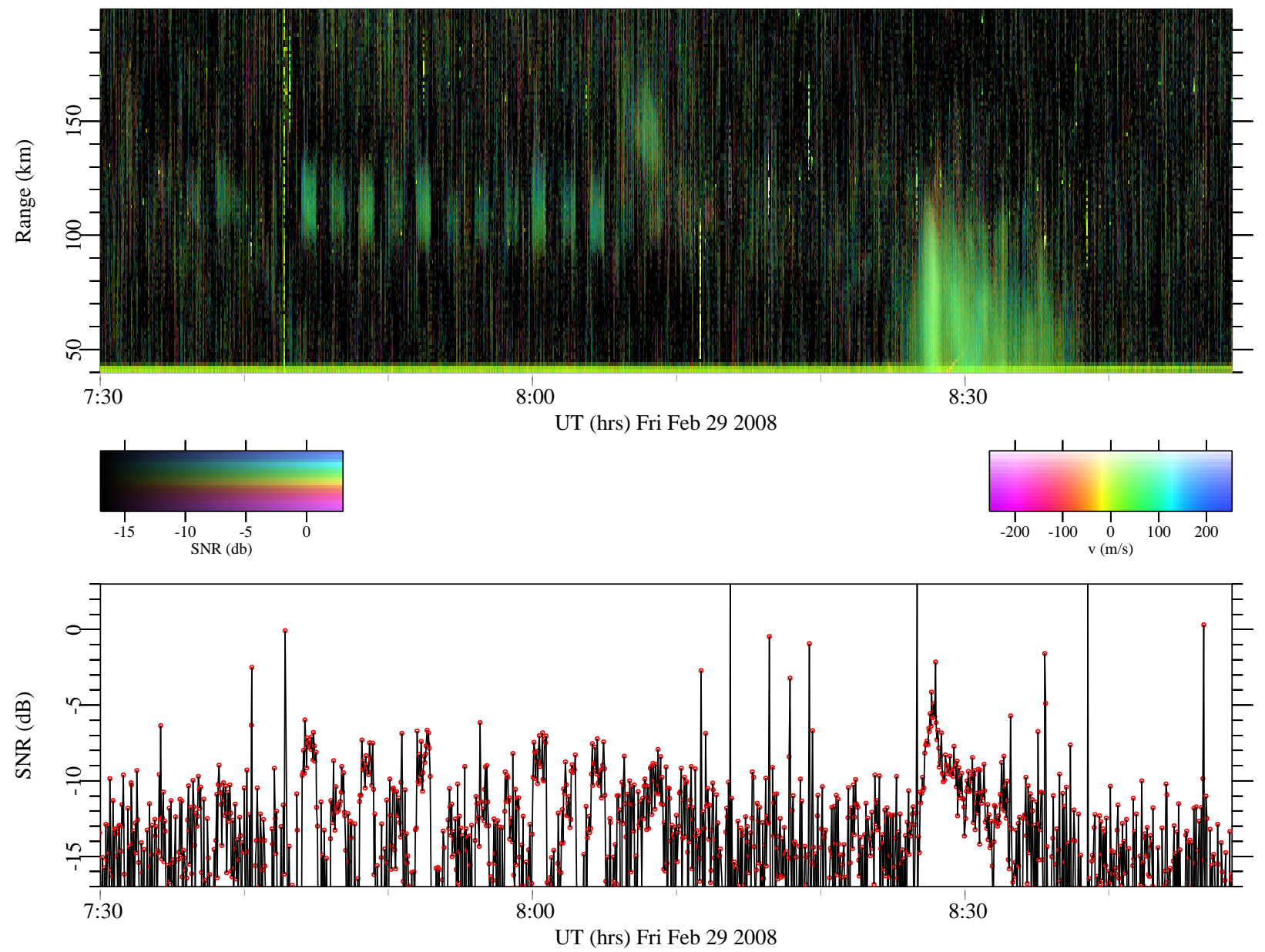

Fig. 3. RTDI plot of backscatter from artificial E region FAIs over HAARP observed on 29 February 2008, during a sporadic E layer event. Natural auroral echoes are evident in apparent ranges outside the $90-140 \mathrm{~km}$ interval.The incoherent integration time for the figure is about $13 \mathrm{~s}$.

gyroharmonic frequency. Small changes in echo range and intensity were reported, but these are no larger than the kind of secular variations routinely observed and attributed to background density profile variability and the sensitivity of the thermal oscillating two-stream instability to the height of the upper hybrid resonance layer with respect to the pump standing wave. Other diagnostics unavailable for these experiments may do so, but the coherent scatter radar does not clearly distinguish between irregularities generated with pump frequencies above and below $2 \Omega_{e}$. As hysteresis effects indicative of wave trapping are evident in experiments involving pump frequencies below $2 \Omega_{e}$ (Nossa et al., 2009), theory governing wave trapping in this frequency regime warrants additional investigation.

Following McBride (1970) and Huang and Kuo (1994), we seek a kinetic theory of upper hybrid waves in a weakly ionized plasma that includes the effects of finite parallel wavenumbers. An appropriate expression for the longitudinal dielectric permittivity for a magnetized Maxwellian elec- tron plasma incorporating the BGK collisional integral has been derived by Alexandrov et al. (1984) (see also Mjølhus (1993) and Kryshtal (1998) for notational guides):

$$
\begin{aligned}
& \epsilon(\omega, \mathbf{k}) \\
& =\frac{k_{i} k_{j}}{k^{2}} \epsilon_{i j}(\omega, \mathbf{k}) \\
& \alpha_{n}=\left(\omega+i v-n \Omega_{e}\right) /\left(\left|k_{\|}\right| \sqrt{T / m}\right) \\
& \Lambda_{n}(z)=I_{n}(z) e^{-z} \\
& \chi^{2}=k_{\perp}^{2} K T / m \Omega_{e}^{2} \\
& =k_{\perp}^{2} \rho^{2}
\end{aligned}
$$$$
=1+\frac{k_{d}^{2}}{k^{2}}\left\{\frac{1+\sum_{n=-\infty}^{\infty} \frac{\omega+i v}{\omega+i v-n \Omega}\left[W\left(\alpha_{n}\right)-1\right] \Lambda_{n}\left(\chi^{2}\right)}{1+\sum_{n=-\infty}^{\infty} \frac{i v}{\omega+i v-n \Omega_{e}}\left[W\left(\alpha_{n}\right)-1\right] \Lambda_{n}\left(\chi^{2}\right)}\right\}
$$

where $\Omega_{e}$ is the electron gyrofrequency, $v$ is the electronneutral collision frequency, $k_{\perp}$ and $k_{\|}$are the perpendicular 


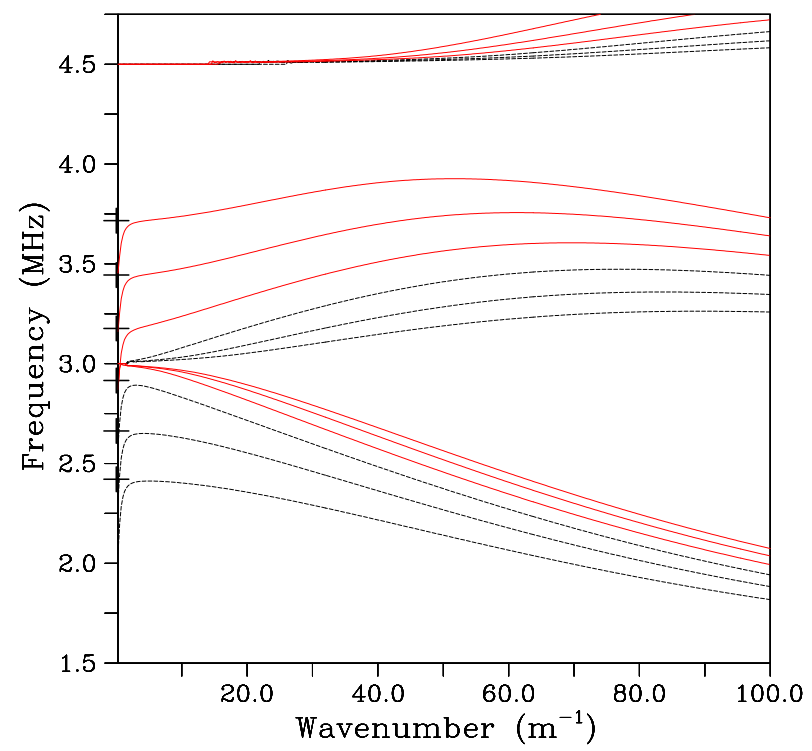

Fig. 4. Dispersion curves for upper hybrid/electron Bernstein waves versus $k_{\perp}$ with $\Omega_{e} / 2 \pi=1.5 \mathrm{MHz}, \nu=5000 \mathrm{~s}^{-1}$, and $k_{\|}=2 \pi / 10 \mathrm{~m}^{-1}$ for six different plasma frequencies $\left(\omega_{p} / 2 \pi \epsilon\right.$ $\{1.9,2.2,2.5,2.8,3.1,3.4\} \mathrm{MHz})$. The corresponding upper hybrid frequencies, $\omega_{\mathrm{uh}} / 2 \pi$, are indicated by + symbols on the ordinate. Dispersion curves corresponding to upper hybrid frequencies below (above) $2 \Omega_{e} / 2 \pi=3 \mathrm{MHz}$ are indicated with black solid (red dashed) lines.

and parallel wavenumbers, respectively, $k_{d} \equiv \lambda_{d}^{-1}$ is the Debye wavenumber, $\rho$ is the electron gyroradius, and where the other symbols have the usual meaning. Also, $I_{n}$ is the modified Bessel function of the first kind of order $n$ and $W$ is the plasma dispersion function, which is related to the complex error function (e.g. Fried and Conte, 1961).

The roots of Eq. (1) can be found numerically with the aid of a few readily available computational tools. These include an efficient plasma dispersion function calculator, a modified Bessel function calculator, and a globally convergent variant of Newton's method (Poppe and Wijers, 1990; Amos, 1986; Powell, 1970). Note that the derivatives of Eq. (1) necessary to populate the Jacobian matrix for the root solver have elementary analytic forms.

The roots of Eq. (1) for frequencies in the vicinity of the second electron gyroharmonic frequency plotted versus transverse wavenumber $k_{\perp}$ are shown in Fig. 4. For the computation, we have taken $\Omega_{e} / 2 \pi=1.5 \mathrm{MHz}, \nu=5000 \mathrm{~s}^{-1}$, $k_{\|}=2 \pi / 10 \mathrm{~m}^{-1}$ and $T=350 \mathrm{~K}$. Curves for six different plasma frequencies, equally spaced between 1.9 and $3.4 \mathrm{MHz}$, are plotted. The corresponding upper hybrid frequencies, $\omega_{\text {uh }} / 2 \pi$, are indicated by + symbols on the ordinate. Three curves corresponding to conditions with upper hybrid frequencies below (above) the second electron gyroharmonic frequency are plotted using dashed black (solid red) lines.
In the limit $k_{\|} \rightarrow 0$, the dispersion curves remain similar to those plotted in Fig. 4 at large $\chi^{2}$ (short transverse wavelengths). At long transverse wavelengths, however, the curves are cutoff at the upper hybrid frequency $\omega_{\mathrm{uh}}$. All curves in the frequency band below $2 \Omega_{e}$ decrease monotonically in frequency with increasing transverse wavenumber, whereas all curves above $2 \Omega_{e}$ increase in frequency from a low-frequency cutoff, attain a maximum frequency at a second, high-frequency cutoff, and then decrease in frequency once again. Mjølhus (1993) terms the branches to the left and right of the second cutoff upper hybrid and electron Bernstein modes, respectively.

Wave trapping can be investigated using plots like Fig. 4 through eikonal analysis (e.g. Landau and Lifshitz, 1987; Weinberg, 1962), noting that wave packets will propagate in an inhomogeneous medium in such a way that frequency remains invariant provided that the medium itself is not changing rapidly. A wave packet launched at the low-frequency cutoff of an upper hybrid mode travels along a horizontal line in Fig. 4 as it penetrates into a depleted caviton. Propagating into regions of lower density, it will ultimately encounter a turning point at the trough and be reflected, all the time remaining on viable dispersion curves. A measure of the maximum depth of the caviton that can be formed through the resonance instability is the difference in the two cutoff frequencies of a given dispersion curve, which is related to the maximum difference in plasma number densities at the center and walls of the deepest caviton accessible to the wave packet. Above $2 \Omega_{e}$, this difference decreases as the upper hybrid frequency approaches an electron gyroharmonic from below (Mjølhus, 1993). Since there are no second cutoffs in curves in the frequency band below $2 \Omega_{e}$, trapping cannot occur, i.e., wave packets launched at the low-frequency cutoff cannot enter depleted cavitons and remain simultaneously on a viable dispersion curve and a surface of constant frequency. Such waves would be excluded from cavitons rather than trapped within them in the $k_{\|} \rightarrow 0$ limit.

The inclusion of finite parallel wavenumbers in the calculation changes the picture described above by introducing or modifying the low-frequency cutoff in the dispersion curves at small transverse wavenumbers. The shapes of the curves in the small $k_{\perp}$ limit depend on the size of $k_{\|}^{2}$ as well as on the collision frequency. However, the long perpendicular wavelength cutoff is a consequence neither of collisional nor collisionless damping and instead mainly reflects the reduced restoring force imposed by the magnetic field for waves propagating obliquely to $\mathbf{B}$. Dispersion curves otherwise approaching the upper hybrid frequency at long transverse wavelengths dip sharply in frequency as a result in all gyroharmonic frequency bands. From the point of view of eikonal analysis, wave trapping therefore does not appear to be prohibited at frequencies below the second electron gyroharmonic frequency when finite parallel wavenumbers are permitted. As the high-frequency cutoff occurs at relatively small values of $k_{\perp}$ for waves in the frequency band below 
$2 \Omega_{e}$ and for those just below $2 \Omega_{e}$ in particular, trapping may be limited to waves with relatively small transverse wavelengths in this band, with the limiting value related to the size of $k_{\|}$.

Additional insights can be obtained by considering a simplified, algebraic form of the dispersion relation. We will regard $k_{\|}$as being sufficiently small to permit the approximation that, with $\omega-n \Omega_{e} \neq 0, a_{n} \rightarrow \infty$, and $W\left(a_{n}\right) \rightarrow 0$. This limit excludes Landau (cyclotron) damping and reduces the dispersion relation in Eq. (1) to:

$\epsilon(\omega, \mathbf{k})=1+\frac{k_{d}^{2}}{k^{2}}\left\{\frac{1-\sum_{n=-\infty}^{\infty} \frac{\omega+i v}{\omega+i \nu-n \Omega_{e}} \Lambda_{n}\left(\chi^{2}\right)}{1-\sum_{n=-\infty}^{\infty} \frac{i \nu}{\omega+i v-n \Omega_{e}} \Lambda_{n}\left(\chi^{2}\right)}\right\}$

The sums in this formula can be further simplified with the following identities:

$$
\begin{aligned}
I_{n}(z) & =I_{-n}(z) \\
1 & =\sum_{n=-\infty}^{\infty} \Lambda_{n}(z)
\end{aligned}
$$

with the result

$\epsilon(\omega, \mathbf{k})$

$$
=1-2 \omega_{p}^{2} \frac{k_{\perp}^{2}}{k^{2}} \frac{e^{-\chi^{2}}}{\chi^{2}} \frac{\omega+i \nu}{\omega} \sum_{n=1}^{\infty} \frac{n^{2} I_{n}\left(\chi^{2}\right)}{(\omega+i \nu)^{2}-n^{2} \Omega_{e}^{2}}
$$

where only the $n=0$ term in the denominator of Eq. (2) has been retained. We next consider an expansion of Eq. (3) in orders of the $\chi^{2}$ parameter, focusing on behavior at long transverse wavelengths and making use of the following expansion for $I_{n}$ :

$I_{n}(z)=\left(\frac{1}{2} z\right)^{n} \sum_{k=0}^{\infty} \frac{\left(\frac{1}{4} z^{2}\right)^{k}}{k ! \Gamma(n+k+1)}$

The zero and first order terms in the dispersion relation can then be shown to be:

$$
\begin{aligned}
& \mathcal{O}\left(\chi^{0}\right): 1-\frac{k_{\perp}^{2}}{k^{2}} \frac{X(1+i Z)}{(1+i Z)^{2}-Y^{2}} \\
& \mathcal{O}\left(\chi^{2}\right):-\chi^{2} \frac{k_{\perp}^{2}}{k^{2}} \frac{3 X(1+i Z) Y^{2}}{\left((1+i Z)^{2}-Y^{2}\right)\left((1+i Z)^{2}-4 Y^{2}\right)}
\end{aligned}
$$

where we make use of the standard notation $X \equiv \omega_{p}^{2} / \omega^{2}$, $Y \equiv \Omega_{e} / \omega$, and $Z \equiv v / \omega$ and consider only the $n=1$ and $n=2$ terms in the sum in Eq. (3). Note that investigating asymmetric collisionless damping above and below gyroharmonic frequencies necessitates retaining higher order terms in $\chi^{2}$ as well as larger values of $n$ in the sum. We truncate here to maintain consistency with other work, focusing on the effects of finite parallel wavenumbers and relegating asymmetry to future analysis.
Combining the components above yields the following, fluid-theory approximation for the dielectric permittivity:

$\epsilon(\omega, k)$

$=1-\frac{k_{\perp}^{2}}{k^{2}} \frac{X}{1-Y^{2}}\left(1+i Z \frac{1+Y^{2}}{1-Y^{2}}+\frac{3 \chi^{2} Y^{2}}{1-4 Y^{2}}\right)$

In calculating Eq. (4), we regard the second and third terms in the parentheses as small corrections associated with collisional and thermal effects, respectively, and neglect additional cross terms combining both effects. The $\epsilon=0$ result is then consistent with the dispersion relations analyzed by Mjølhus (1993) and Kosch et al. (2007), for example, in the appropriate limiting regimes.

Dysthe et al. (1982) and then Mjølhus (1993) used dielectric permittivity functions similar to Eq. (4) to develop ordinary differential equations describing upper hybrid wave trapping in depleted cavitons in one dimension transverse to the geomagnetic field. They did this by linearizing Gauss' law for dielectric media, $\nabla \cdot(\epsilon \mathbf{E})=0$, where the electric field includes the electromagnetic pump field and the electrostatic response. The linearization introduced an additional small correction associated with the density perturbation of the caviton. Equation (4) adds a fourth correction due to finite parallel wavenumbers. Following the formalism of the earlier works and including the finite $k_{\|}$correction, which is formulated somewhat differently here than by Dysthe et al. (1982), we can derive the following coupled system of differential equations describing the transverse electric field of the upper hybrid wave $E_{1}$ and an auxiliary field $F_{1}$ :

$$
\begin{aligned}
F_{1}+\lambda^{2} \frac{d^{2} E_{1}}{d x^{2}}+\left(\epsilon_{0}-i \delta-N(x)\right) E_{1} & =p E_{\circ} N(x) \\
\left(\frac{-d^{2}}{d x^{2}}+k_{\|}^{2}\right) F_{1} & =k_{\|}^{2} E_{1}
\end{aligned}
$$

where $x$ is the direction transverse to the geomagnetic field, $E_{\circ}$ represents the pump mode field amplitude, and where the other symbols are defined by

$$
\begin{aligned}
\lambda^{2} & =3 \rho^{2} Y^{2} /\left(1-4 Y^{2}\right) \\
\epsilon_{0} & =1-X /\left(1-Y^{2}\right) \\
\delta & =Z\left(1+Y^{2}\right) /\left(1-Y^{2}\right) \\
N & =\delta n / n_{\circ} \\
p E_{\circ} N(x) & =-\hat{k} \cdot \delta \epsilon \cdot \mathbf{E}_{\circ}
\end{aligned}
$$

with $p$ a geometric factor.

Figure 5 shows representative solutions of Eqs. (5) and (6) (with $E_{1}(x)$ plotted here) demonstrating wave trapping. The top panel represents $\epsilon_{0}-N(x)$ for a depleted caviton and illustrates how the dielectric constant is positive (negative) inside (outside) the depletion. Neglecting the terms involving $k_{\|}^{2}$, Eqs. (5) and (6) represent a second-order differential equation, which is expected to exhibit oscillating 


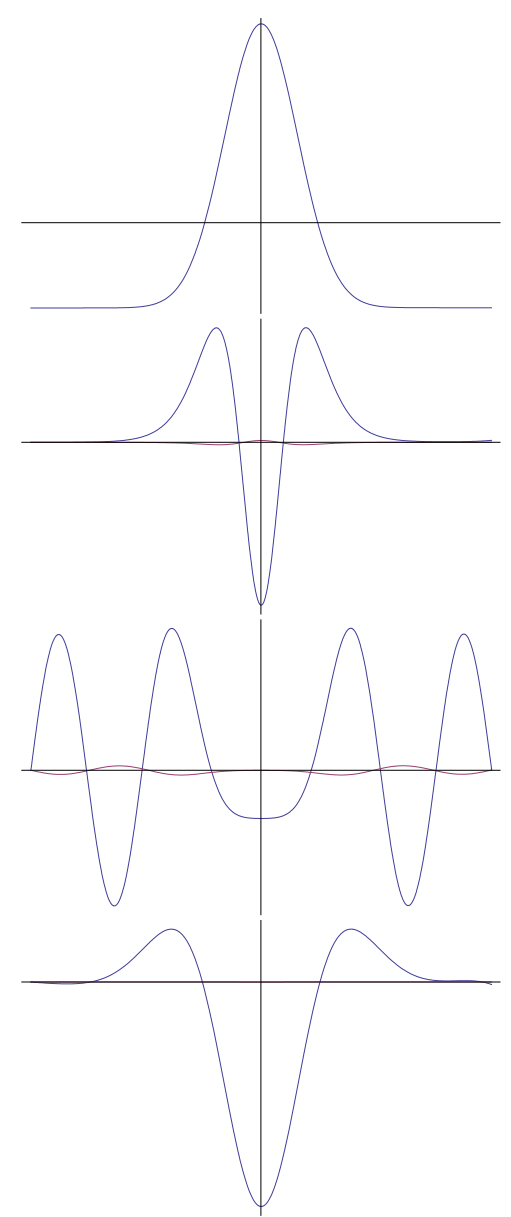

Fig. 5. Top panel: Functional form of $\epsilon_{0}-N(x)$ corresponding to a depleted caviton. Second panel: Solution for the case $k_{\|}^{2}=0$ and $\lambda^{2}>0$. Third panel: Solution for the case $k_{\|}^{2}=0$ and $\lambda^{2}<0$ Bottom panel: Solution for the case $k_{\|}^{2}>0$ and $\lambda^{2}<0$. Dirichlet boundary conditions are applied at either end of the abscissa.

and evanescent behavior inside and outside the depleted region, respectively, provided $\lambda^{2}>0$ and $\delta \ll 1$. This implies wave trapping inside the caviton, as illustrated by the second panel of Fig. 5. The number of waveforms contained within the depletion depends on the size of $\lambda^{2}$ relative to the size of $\epsilon_{0}-N(x)$. When the parameter controlling $\epsilon_{0}-N(x)$ approaches an eigenvalue of the homogeneous system of equations, the solutions grow without bound. This is the nature of the resonance instability, with actual resonances corresponding to integral numbers of waveforms fitting precisely into the depleted zone. Various limiting cases of the problem have been described in detail by Dysthe et al. (1982) and Mjølhus (1993).

The third panel of Figure 5 shows a representative solution for the $\lambda^{2}<0$ case, which applies when $\omega<2 \Omega_{e}$. Here, the situation is reversed, such that the wave is evanescent within and therefore excluded from the caviton. The caviton will experience a deficit of wave heating compared to its surroundings in this case, and the resonance instability will not occur. Note that trapped solutions can reemerge if large values of $\delta$ are considered, although dissipation associated with collisional damping will tend to be stabilizing.

Finally, the bottom panel of Fig. 5 shows a representative solution to Eqs. (5) and (6) for the case $\lambda^{2}<0$ but with the $k_{\|}^{2}$ terms restored this time. The resulting, fourth-order differential equation is seen to be capable of producing trapped solutions in the $\omega<2 \Omega_{e}$ regime. The morphology of the solution, number of waveforms in the depletion, etc., are controlled by the ratios of $k_{\|}^{2}, \lambda^{2}$, and $\epsilon_{0}-N(x)$ in a more complicated way than was the second-order system. The boundary between oscillating and evanescent wave behavior depends not only on the sign of $\epsilon$ but also on its curvature, for example.

\section{Discussion}

Sustained critical frequency (FoE) values of about $3 \mathrm{MHz}$ during geomagnetically quiet summer afternoons afford the possibility of routine $\mathrm{E}$ region ionospheric modification experiments over HAARP, which is able to operate at frequencies as low as $2.75 \mathrm{MHz}$ in practice. Absent geomagnetic activity, the experiments are relatively immune to variations in heating and propagation conditions that can interfere with the consistency of $\mathrm{F}$ region experiments. Artificial E region FAIs can be monitored using the coherent scatter radar near Homer, the result being a well controlled experiment. Most of the experiments performed so far were undertaken at heating frequencies below the second electron gyroharmonic. Preliminary results argue that the thermal OTSI, wave trapping, and the resonance instability are at work at these experiments (Nossa et al., 2009).

The radar experiments described here do not differentiate clearly between heating phenomena taking place at pump frequencies below and above the second electron gyroharmonic frequency in the main. Upper hybrid wave trapping in density cavitons therefore does not appear to be ruled out at pump frequencies below $2 \Omega_{e}$. Analysis suggests that a second, low-frequency cutoff in the upper hybrid wave dispersion relation, which is required for wave trapping, can be introduced at long perpendicular wavelengths by including the effects of finite parallel wavenumbers. Below $2 \Omega_{e}$, the high-frequency cutoff occurs at a relatively small transverse wavenumber that depends on the value of $k_{\|}$. This suggests that the trapped waves may not be able to attain very short transverse wavelengths below $2 \Omega_{e}$, and that some heatingrelated phenomena may therefore be interrupted, even if 5-m scale FAI generation is not.

A number of studies, including those by Dysthe et al. (1982) and Mjølhus (1993), considered finite parallel wavenumber effects, focusing mainly on Landau damping, and the associated suppression of upper hybrid waves in 
the immediate vicinity of electron gyroharmonic frequencies. The condition for neglecting Landau damping at all $\chi^{2}$ is that $\omega-n \Omega_{e} \gg\left|k_{\|}\right| v_{t e}$. (A less restrictive condition was also derived for the limit of small $\chi^{2}$.) In view of the fact that we have not as a rule observed wave suppression within $\sim 50 \mathrm{kHz}$ of the second electron gyroharmonic frequency, this implies an upper bound of about $k_{\|}=4 \mathrm{~m}^{-1}$ in the E region, which is an order of magnitude larger than the value used to compute Figure 4 (where the effects of $k_{\|}$were accentuated for plotting purposes) and 40 times the value used to compute the bottom panel of Fig. 5. Evaluating experimental and theoretical evidence from a number of sources, Mjølhus (1993) found upper limits on $k_{\|}$of order unity in MKS units in the $F$ region. A reasonable expectation in practice might be for $k_{\|}$to match approximately the wavenumber of the pump wave. In the future, it may be possible to estimate the parallel wavenumber from observations of FAI suppression at pump frequencies in the stop band closer to the electron gyroharmonic. Measuring a frequency crossing in the $\mathrm{E}$ region is somewhat more difficult than it is in the $\mathrm{F}$ region given the relatively small altitude excursion of the $\mathrm{E}$ layer during the daytime (see Kosch et al., 2007, for reference). Sporadic E layers may pose a more suitable target for this kind of experiment, although the disturbed conditions that often accompany them at high latitudes undermine the controlled nature of the experiment.

Radar interferometry is routinely used to measure the aspect sensitivity of FAIs in the ionosphere (e.g. Farley et al., 1981). The results from such experiments generally refer to the RMS deviation or spreading of the scattering wavevector about the normal direction, which is easy to measure, rather than a systematic bias in a given direction, which is not. We might equally well interpret the parallel wavenumber in question here as an RMS deviation about the normal direction. The mathematics is essentially identical, although the physical picture is different. However, eikonal analysis in the vertical (parallel to B) direction also suggests that the upper hybrid waves should acquire a bias toward finite $k_{\|}$ while propagating, even if launched transverse to $\mathbf{B}$, suggesting that a combination of bias and spread in $k_{\|}$could be present. Note that, as was pointed out by Mjølhus (1993), the parallel wavenumber of the upper hybrid wave is not the same thing as the parallel wavenumber of the striations visible to radar, and so radar interferometry cannot by itself resolve the role or interpretation of parallel wavenumbers in this context. Note also that it is the aspect sensitivity of the striations that concerned Grach (1979) in his calculation of the threshold for thermal parametric instability.

Although the data quality is marginal, our sole example of artificial FAIs generated in a sporadic E layer suggests that wave trapping at pump frequencies below $2 \Omega_{e}$ can be suppressed under the right circumstances. The ionogram for the event in question placed the sporadic $\mathrm{E}$ layer at about $110 \mathrm{~km}$ altitude, close to the critical height in the daytime. We therefore cannot conclude that either pump frequency was closer to $2 \Omega_{e}$ than in the daytime experiments. Given that the upper-hybrid interaction height in a sharply-peaked sporadic E layer is apt to be extraordinarily narrow, we may speculate that parallel wavenumbers may be drastically limited by the requirement that the trapped waves may not propagate vertically out of the layer. This imposes an additional limit on the shortest transverse wavelength for which trapping can occur. It could be that wave trapping at pump frequencies below $2 \Omega_{e}$ either cannot occur in a narrow sporadic E layer or cannot excite cavitons sufficiently small to appear as strong targets to a VHF radar. Further experiments involving additional diagnostics are needed to elucidate this behavior.

Acknowledgements. The authors are grateful for help received from the NOAA Kasitsna Bay Laboratory, its Director Kris Holderied, Lab Manager Mike Geagel, and Lab Director Connie Geagel. We also appreciate helpful comments from C. La Hoz from the University of Troms $\emptyset$ who is presently on leave at Cornell. This work was supported by the High Frequency Active Auroral Research Program (HAARP) and by the Office of Naval Research and the Air Force Research Laboratory under grant N00014-07-1-1079 to Cornell.

Topical Editor M. Pinnock thanks A. Streltsov and B. Isham for their help in evaluating this paper.

\section{References}

Alexandrov, A. F., Bogdankevich, L. S., and Rukhadze, A. A.: Principles of Plasma Electrodynamics, Springer Verlag, New York, 1984.

Amos, D. E.: A portable package for Bessel functions of a comples argument and nonnegative order, Trans. Math. Software, 12, 265-273, 1986.

Das, A. C. and Fejer, J. A.: Resonance instability of small-scale field-aligned irregularities, J. Geophys. Res., 84, 6701-6704, 1979.

Djuth, F. T., Pedersen, T. R., Gerkin, E. A., Bernhardt, P. A., Selcher, C. A., Bristow, W. A., and Kosch, M. J.: Ionospheric modification at twice the electron cyclotron frequency, Phys. Rev. Lett., 94, 125,001-1-4, 2005.

Djuth, F. T., Jost, R. J., Noble, S. T., Gordon, W. E., Stubbe, P., Kopka, H., Nielsen, E., Boström, R., Derblom, H., Hedberg, Á., and Thidé, B.: Observations of $E$ region irregularities generated at auroral latitudes by a high-power radio wave, J. Geophys. Res., 90, 12293-13206, 1985.

Dysthe, K., Mjølhus, E., Pécseli, H., and Rypdal, K.: Thermal cavitons, Phys. Scr. T., 2, 548-559, 1982.

Dysthe, K., Mjølhus, E., Pécseli, H., and Rypdal, K.: A thermal oscillating two-stream instability, Phys. Fluids, 26, 146-157, 1983.

Farley, D. T., Ierkic, H. M., and Fejer, B. G.: Radar interferometry: A new technique for studying plasma turbulence in the ionosphere, J. Geophys. Res., 86, 1467-1472, 1981.

Fejer, J. A.: Ionospheric modification and parametric instabilities, Rev. Geophys. Space Phys., 17, 135-153, 1979.

Fialer, P. A.: Field-aligned scattering from a heated region of the ionosphere - Observations at HF and VHF, Radio Sci., 9, 923940, 1974. 
Fried, B. D. and Conte, S. D.: The Plasma Dispersion Function and Hilbert Transform of the Gaussian, Academic Press, 1961.

Frolov, V. L., Erukhimov, L. M., Metelev, S. A., and Sergeev, E. N.: Temporal behavior of artificial small-scale ionospheric irregularities: review of experimental results, J. Atmos. Sol. Terr. Phys., 18, 2317-2333, 1997.

Grach, S., Mityakov, N., Rapoport, V., and Trakhtengertz, V.: Thermal parametric turbulence in a plasma, Physica, D, 2, 102-106, 1981.

Grach, S. M.: Thermal parametric instability in ionospheric plasma at frequencies close to $\omega_{\mathrm{He}}$ and $2 \omega_{\mathrm{He}}$, Radiophys. Quantum Electron., 22, 357-363, 1979.

Grach, S. M., Karashtin, A. N., Mityzkov, N. A., Rapoport, V. O., and Trakhtengerts, V. Y.: Theory of thermal parametric instability in an inhomogenous plasma, Sov. J. Plasma Phys. Engl. Transl., 4, 737-741, 1978.

Gurevich, A. V.: Nonlinear effects in the ionosphere, Uspekhi Fizicheskikh Nauk., 177(11), 1145-1177, 2007.

Haslett, J. C. and Megill, L. R.: A model of the enhanced airglow excited by RF radiation, Radio Sci., 9, 1005-1019, 1974.

Honary, F., Robinson, T. R., Wright, D. M., Stocker, A. J., Rietveld, M. T., and McCrea, I.: Letter to the Editor: First direct observations of the reduced striations at pump frequencies close to the electron gyroharmonics, Ann. Geophys., 17, 1235-1238, 1999, http://www.ann-geophys.net/17/1235/1999/.

Huang, J. and Kuo, S.: Cyclotron harmonic effect ion the thermal oscillating two-stream instability in the high latitude ionosphere, J. Geophys. Res., 99, 2173-2181, 1994.

Hysell, D. L.: $30 \mathrm{MHz}$ radar observations of artificial $\mathrm{E}$ region field-aligned plasma irregularities, Ann. Geophys., 26, 117-129, 2008, http://www.ann-geophys.net/26/117/2008/.

Inhester, B., Das, A. C., and Fejer, J. A.: Generation of small-scale field-aligned irregularities in ionospheric heating experiments, J. Geophys. Res., 86, 9101-9105, 1981.

Istomin, Y. N. and Leyser, T. B.: Electron acceleration by cylindrical upper hybrid oscillations trapped in density irregularities in the ionosphere, Phys. Plasmas, 10, 2962-2970, 2003.

Kosch, M. J., Rietveld, M. T., Kavanagh, A. J., Davis, C., Yeoman, T., Honary, F., and Hagfors, T.: High-latitude pumpinduced optical emissions for frequencies close to the third electron gyro-harmonic, Geophys. Res. Lett., 29(23), 2112, doi:10.1029/2002GL015744, 2002.

Kosch, M. J., Pedersen, T., Hughes, J., Marshall, R., Gerken, E., Senior, A., Sentman, D., McCarrick, M., and Djuth, F. T.: Artificial optical emissions at HAARP for pump frequencies near the third and second electron gyro-harmonic, Ann. Geophys., 23, 15851592, 2005, http://www.ann-geophys.net/23/1585/2005/.

Kosch, M. J., Pedersen, T., Mishin, E., Oyama, S., Hughes, J., Senior, A., Watkins, B., and Bristow, B.: Coordinated optical and radar observations of ionospheric pumping for a frequency pass through the second electron gyroharmonic at HAARP, J. Geophys. Res., 112, A06325, doi:10.1029/2006JA012,146, 2007.

Kryshtal, A. N.: Bernetein-wave instability in a collisional plasma with a quasistatic electric field, J. Plasma Physics, 60, 469-484, 1998.
Kuo, S. P. and Lee, M. C.: On the parametric excitation of plasma modes at upper hybrid resonance, Phys. Lett. A, 91, 444-446, 1982.

Landau, L. D. and Lifshitz, E. M.: The Classical Theory of Fields, Pergamon Press, New York, 1987.

Lee, M. C. and Kuo, S. P.: Excitation of upper hybrid waves by a thermal parametric instability, J. Plasma Phys., 30, 463-478, 1983.

McBride, J. M.: Parametric excitation of purely growing mode in magneto-plasmas, Phys. Fluids, 13, 2725-2727, 1970.

Minkoff, J., Kugelman, P., and Weissman, I.: Radio frequency scattering from a heated ionospheric volume, 1, VHF/UHF fieldaligned and plasma line backscatter measurements, Radio Sci., 9, 941-955, 1974.

Mishin, E. V., Kosch, M. J., Pedersen, T. R., and Burke, W. J.: HFinduced airglow at magnetic zenith: Thermal and parametric instabilities near electron gyroharmonics, Geophys. Res. Lett., 32, L23106, doi:10.1029/2005GL023864, 2005.

Mjølhus, E.: On linear conversion in magnetized plasmas, Radio Sci., 6, 1321-1339, 1990.

Mjølhus, E.: On the small scale striation effect in ionospheric modification experiments near harmonics of the electron gyro frequency, J. Atmos. Terr. Phys., 55(6), 907-918, 1993.

Noble, S. T., Djuth, F. T., Jost, R. J., Gordon, W. E., Hedberg, A., Thide, B., Derblom, H., Bostrom, R., Nielsen, E., Stubbe, P., and Kopka, H.: Multiple-frequency radar observations of highlatitude $E$ region irregularities in the HF modified ionosphere, J. Geophys. Res., 92, 13613-13627, 1987.

Nossa, E., Hysell, D. L., Fallen, C. T., and Watkins, B. J.: Radar observations of artificial E-region field-aligned irregularities, Ann. Geophys., 27, 2699-2710, 2009, http://www.ann-geophys.net/27/2699/2009/.

Ponomarenko, P. V., Leyser, T. B., and Thidé, B.: New electron gyroharmonic effects in the HF scatter from pump-excited magnetic field-aligned ionospheric irregularities, J. Geophys. Res., 104, 10081-10087, 1999.

Poppe, G. P. M. and Wijers, C. M. J.: More efficient computation of the complex error function, Trans. Math. Software, 16, 38-46, 1990.

Powell, M. J. D.: A hybrid method for nonlinear equations, in: Numerical Methods for Nonlinear Algebraic Equations, edited by: Rabinowitz, P., pp. 87-114, Gordon and Breach, London, 1970.

Robinson, T. R.: The heating of the high latitude ionosphere by high power radio waves, Phys. Rep., 179(2), 79-209, 1989.

Stubbe, P.: Review of ionospheric modification experiments at Tromsø, J. Atmos. Terr. Phys., 59, 349-368, 1996.

Vas'kov, V. V. and Gurevich, A. V.: Resonance instability of smallscale plasma perturbations, Sov. Phys. JETP Engl. Trans., 46, 487-494, 1977.

Weinberg, S.: Eikonal method in magnetohydrodynamics, Phys. Rev., 126, 1899-1909, 1962. 